\title{
Commensurate monolayers on surfaces: Geometry and ground states
}

\author{
Alexandre Tkatchenko* \\ Departamento de Química, Universidad Autónoma Metropolitana-Iztapalapa, San Rafael Atlixco 186, Vicentina, \\ Apartado Postal 55-534, México, Distrito Federal 09340, Mexico
}

(Received 24 April 2007; published 7 June 2007)

\begin{abstract}
We present a procedure for the generation of all rigid commensurate monolayer-surface structures of a given symmetry, up to a certain number of adsorbate particles, $N_{a d s}$, in the unit cell. It is shown that the minimum energy structures in each unit cell are related to a well-defined sequence of Fourier terms of the single-particlesurface potential. This fact allows the prediction of stable commensurate monolayer-surface structures with the only knowledge of Fourier coefficients of the atom-surface potential. The impact of the presented theory for theoretical and experimental determination of atomic and molecular monolayer ground states, as well as its extension to higher dimensions (i.e., for intercalated crystals), is discussed.
\end{abstract}

DOI: 10.1103/PhysRevB.75.235411

PACS number(s): 68.35.-p, 61.14.Hg, 68.43.Fg

\section{INTRODUCTION}

From all the different types of structures identified up to date for two-dimensional monolayer-surface systems, ${ }^{1-3}$ the commensurate ones are particularly interesting because of their potential for technological applications ${ }^{4}$ and their beautiful symmetry, as can be appreciated from the scanning tunneling microscopy experiments. ${ }^{1,4-6}$ The commensurate structures are characterized as having a defined repeating unit cell and their appearance is thought to be governed by the strong adsorbed-particle-surface interaction. The formation of well-ordered commensurate domains has been observed by many experimental techniques for atomic and molecular adsorptions alike. ${ }^{7}$ Figure 1 illustrates the models for two different rigid hexagonal commensurate monolayer arrangements frequently found in experiments. The analysis of this paper will be centered around this type of structure.

The low energy electron diffraction (LEED) technique is frequently employed for the determination of the adsorbed monolayer symmetry and continues to be very helpful for identification of different monolayer structures. ${ }^{1-3}$ The standard LEED approach does not allow one to determine the translational symmetry of the monolayer (center-of-mass position), and extensions of the technique (i.e., dynamic LEED) have been used to solve this difficult problem. However, the determination of the exact translational symmetries is a highdimensional problem which requires an initial guess of atomic positions as an input, therefore making such an approach biased. Even for one atom in the unit cell, the determination of the most stable adsorption site can pose severe difficulties, as it is evident from the long history of investigation of the most stable adsorption site for $\mathrm{Xe}$ on the Pt(111) surface. ${ }^{9}$

The identification of stable commensurate arrangements of a given monolayer-surface system is a highly relevant problem for basic science and technological applications as well. In this work, the procedure which can generate all rigid commensurate structures characterized by $N_{a d s}<\infty$ adsorbate and $N_{\text {sub }}$ substrate particles is presented. Assuming that the monolayer adapts the symmetry of the underlying surface, one can exactly determine all the possible commensurate monolayer structures (the monolayer interatomic spacing, ro- tation angle, and center-of-mass position) by only having the Fourier amplitudes for the single-particle-surface potential. The presented approach can aid the experimentalists and theoreticians in search for the most probable commensurate structures when only limited information (the particlesurface potential or experimental observation of a certain structure) is available.

In this work, we are concerned with rigid monolayers of hexagonal ${ }^{10}$ symmetry on (111) surfaces. Other monolayer or surface symmetries could be easily handled by the same approach. In reality, the most interesting surface symmetries are the (111) and (100) ones, as they are frequently stable and do not reconstruct under usual experimental conditions for many elements in the Periodic Table. Although the rigidity constraint would seem to be unjustifiable in general, there is a wide variety of systems where the monolayer arrangement is close enough to the perfectly rigid case. ${ }^{11-14}$ For further discussion about the validity of rigid hexagonal approximation for real systems, see, e.g., Ref. 15.
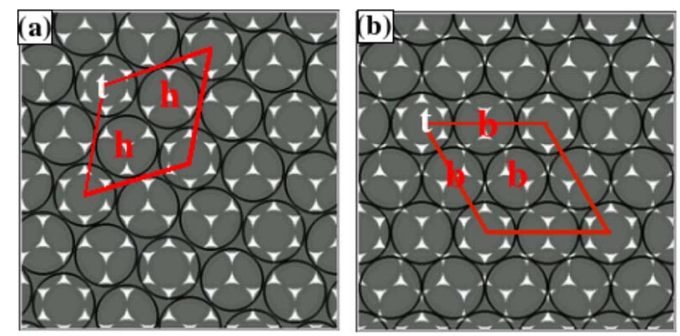

FIG. 1. (Color online) Illustration of two commensurate monolayer-surface structures. (a) $(\sqrt{7} \times \sqrt{7}) R 19.1^{\circ}$ structure with three-atom basis and (b) $(3 \times 3)$ structure with four-atom basis. The filled gray circles indicate the substrate atoms [(111) surface], while bigger black circles show adsorbed monolayer atoms. The unit cell is marked by a red (dark gray) rhomb in both cases. The labels indicate different adsorption sites. In the case of $(\sqrt{7} \times \sqrt{7}) R 19.1^{\circ}$ structure, the unit cell contains one top site and two hollow sites, while $(3 \times 3)$ contains one atom positioned on the top site and three atoms in bridge sites. The pictures were generated by the ALSA software, see Ref. 8. 


\section{GEOMETRY OF COMMENSURATE MONOLAYERS}

The primitive cell of the (111) surface with atomic diameter $d=1$ is spanned by two vectors, $v_{1}=(1,0)$ and $v_{2}$ $=(0.5,0.5 \sqrt{3})$, with $60^{\circ}$ angle between them. The distance from a fixed origin placed at $(x, y)=(0,0)$ to any atom in the hexagonal lattice is given by $D=\sqrt{a^{2}+b^{2}+a b}$, where $a$ and $b$ are integers. The reciprocal lattice of a two-dimensional hexagonal arrangement is another hexagonal arrangement rotated by $30^{\circ}$. Therefore, the length of the reciprocal lattice vectors is written as $D G_{0}$, where $G_{0}=\frac{4 \pi}{\sqrt{3}}$ is the length of the smallest reciprocal vector. $D^{2}$ is an integer and forms the so-called hexagonal number sequence. Any expanded nonprimitive cell of the hexagonal lattice is then obtained as a linear combination of primitive (111) vectors, $V_{1}=(a$ $+0.5 b,-0.5 \sqrt{3} b)$ and $V_{2}=(0.5 a+b, 0.5 \sqrt{3} a)$, where $a$ and $b$ are integers. Interestingly, the number of atoms in such expanded cell is also given by $N=a^{2}+b^{2}+a b$. To show this, note that the area of a hexagonal unit cell is enclosed by a rhomb, with area equal to $A_{r}=0.5 \sqrt{3}\left(a^{2}+b^{2}+a b\right)$. On the other hand, an adsorbate atom occupies the area of a circle with $d=1$, given by $\pi / 4$. The density of hexagonal circle arrangement is $\sqrt{3} \pi / 6$, which makes the effective area occupied by circles equal to $A_{c}=0.5 \sqrt{3}$. Dividing $A_{r}$ by $A_{c}$, the hexagonal number sequence is recovered once again. This means, for instance, that a hexagonal cell with two or five particles cannot exist. The first (nonzero) members of the hexagonal number sequence are $1,3,4,7$, etc.

\section{Hexagonal number sequence}

It is important to state several mathematical properties of the hexagonal number sequence. The equation

$$
m=a^{2}+b^{2}+a b
$$

is called a binary quadratic form or Diophantine equation, ${ }^{16}$ and it is studied within number theory. We will call $\mathcal{H}$ the set of all $\mathrm{m}$ 's (ordered in the ascending order and excluding zero). The first important fact is that, up to date, there is no analytic formula establishing one-to-one correspondence between $\mathcal{H}$ and the positive integers $\mathbb{N}^{+}$. However, there is a simple way to find the number of solutions to Eq. (1) for a particular value $m$. It is given by $6 E(m),{ }^{16}$ where $E(m)$ is the difference between divisors of $m$ of the form $3 l+1$ and of the form $3 l+2$. When $E(m) \leqslant 0$ (i.e., for $m=2,5, \ldots$ ), $m$ does not belong to the hexagonal number sequence, whereas for $E(m)>0$, there is a finite number of solutions to Eq. (1). Furthermore, $E(m)$ is not a bounded function, which can be shown considering the case of $m=p^{n}$, where $p$ is a prime $(p \neq 3)$ which belongs to a hexagonal number sequence and $n$ is an integer. In this case, $E(m)=n+1$. In geometric terms, it means that there can be an arbitrarily large number of atoms in the hexagonal lattice, with the distance $D=\sqrt{p^{n}}$ from the origin. For instance, $E(m)=1$ for $m=1,3,4$, but $E(m)=2$ for $m=7$. This fact is very important for the Fourier expansion of the atom-surface potential as $6 E(\mathrm{~m})$ is the number of reciprocal lattice vectors of length $D=\sqrt{m}$. We can also see that the set $\mathcal{H}$ is closed under multiplication, i.e., if $m_{1}, m_{2} \in \mathcal{H} \Rightarrow m_{1} m_{2} \in \mathcal{H}$. In order to prove the last statement, we need to show that $\left(a_{1}^{2}+b_{1}^{2}+a_{1} b_{1}\right)\left(a_{2}^{2}+b_{2}^{2}+a_{2} b_{2}\right)=\left(a_{3}^{2}+b_{3}^{2}\right.$ $\left.+a_{3} b_{3}\right), \forall a_{i} \in \mathbb{N}^{+}$. Indeed, this identity is fulfilled for $a_{3}$ $=a_{1} a_{2}-b_{1} b_{2}$ and $b_{3}=a_{1} b_{2}+b_{1} b_{2}+b_{1} a_{2}$. The closure property will be used later for the generation of commensurate structures with progressively larger number of adsorbate particles.

\section{Commensurate monolayer construction}

Given a hexagonal number sequence, any (high-order) commensurate monolayer-surface (ads-sub) system can be constructed with $N_{s u b}=n^{2}+k^{2}+n k$ and $N_{a d s}=a^{2}+b^{2}+a b$, where $n, k$ and $a, b$ are integers. In this way, a commensurate structure with coverage $\theta=\frac{N_{a d s}}{N_{s u b}}$ is defined except for the center-of-mass position (translational possibility). The monolayer interatomic distance can be calculated as $R=\sqrt{\frac{N_{\text {sub }}}{N_{\text {ads }}}}$. In addition, to match the monolayer with the substrate, the former has to be rotated by a certain angle $\alpha$, which is not unique for a given $N_{a d s}$ and $N_{\text {sub }}$ and can be calculated by imposing the restriction that one adsorbate particle has to coincide with one substrate atom. Once the monolayer $R$ and $\alpha$ are determined, a simple algorithm can be implemented to generate the positions of the atoms in the substrate and adsorbate layers as follows:

(1) Generate the hexagonal number sequence from Eq. (1), obtaining all $m$ 's with the corresponding values of $a$ and b.

(2) Obtain one combination of $n$ and $k$ which generate $N_{\text {sub }}$ and, as a consequence, the extended unit cell vectors $V_{1}=(n+0.5 k,-0.5 \sqrt{3} k)$ and $V_{2}=(0.5 n+k, 0.5 \sqrt{3} k)$, common for the monolayer and the surface.

(3) Calculate all linear combinations of $v_{1}$ and $v_{2}: V_{\text {sub }}$ $=c v_{1}+d v_{2}$ for $-N \leqslant c, d \leqslant N$, with a large $N$. $v_{1}$ and $v_{2}$ are the primitive (111) cell vectors.

(4) For each $V_{s u b}$, check if the $(x, y)$ coordinates lie inside the unit cell rhomb and discard all $V_{\text {sub }}$ vectors which do not satisfy this condition.

(5) Generate the adsorbate layer atomic positions, where every $V_{a d s}$ is given by

$$
\begin{aligned}
V_{a d s}= & {[(c+0.5 d) R \cos \alpha-0.5 \sqrt{3} d R \sin \alpha, 0.5 \sqrt{3} d R \cos \alpha} \\
& +(c+0.5 d) R \sin \alpha],
\end{aligned}
$$

where $c$ and $d$ are integers.

(6) Repeat step (4) for each $V_{a d s}$, instead of $V_{s u b}$.

\section{GROUND STATES OF COMMENSURATE MONOLAYERS ON SURFACES}

In order to identify all possible rigid commensurate monolayer ground states, one needs to have a set of suitable order parameters which describe the monolayer geometry along with a general way of writing down the monolayer energy. The latter can be accomplished by using the Fourier expansion, which allows one to describe any function with a particular symmetry in real space by using the reciprocal lattice vectors (RLVs). ${ }^{17,18}$ In particular, here we will use the Fourier expansion to describe the atom-surface holding potential imposed by a surface with (111) symmetry. In what follows, the Fourier amplitudes will be called $V_{G}$ for a shell 
of reciprocal vectors of length $|G|$. Similarly, the Fourier term associated with a shell of RLVs of length $|G|=D G_{0}$ will be called $\mathcal{F}_{i}$, so that the adsorbed-particle-surface potential is

$$
V(\mathbf{r})=\sum_{i} V_{G_{i}} \mathcal{F}_{i}(\mathbf{r}),
$$

where $i$ is the position of $D^{2}$ in the set $\mathcal{H}$. Let us assume that we know the amplitudes $V_{G}$ of the atom-surface Fourier expansion up to a particular RLV length $|G| \leqslant D_{\text {lim }} G_{0}$ for our system of interest, with $D_{\text {lim }} \in \mathcal{H}$. The energy of the rigid hexagonal monolayer can be expressed in terms of four variables (order parameters): $E_{h e x}=E\left(R, \alpha, x_{0}, y_{0}\right)$, where $R$ and $\alpha$ are the monolayer interatomic distance and rotation angle, respectively, and $\left(x_{0}, y_{0}\right)$ is the position of the corner of the monolayer unit cell on the substrate surface. Therefore, the problem of finding the ground state structures of an infinite rigid monolayer on (111) surface can be written as a minimization of the following energy function

$$
E_{h e x}\left(R, \alpha, x_{0}, y_{0}\right)=\sum_{c=-\infty}^{\infty} \sum_{d=-\infty}^{\infty} V\left(c, d, R, \alpha, x_{0}, y_{0}\right),
$$

where $V\left(c, d, R, \alpha, x_{0}, y_{0}\right)$ gives the value of the Fourier expansion for a particular atom position, and $c$ and $d$ are the integer multipliers for the primitive unit cell vectors [see Eq. (2)].

Let us take the trivial situation of $N_{a d s}=1$. In this case, the energy of the monolayer (composed of a single atom) is simply a sum over $V(\mathbf{r})$, where all the terms contribute. It would be appealing to generalize this argument to cells with $N_{a d s}$ $>1$. To find the minimizers of Eq. (4) for any Fourier term, we rewrite it as energy per atom (see Eqs. (6)-(8) of Ref. $15)$. Then, the $(R, \alpha)$ ground states for Fourier terms associated with reciprocal vectors $G=\left(G_{x}, G_{y}\right)=G_{0}(0.5 \sqrt{3} b, a$ $+0.5 b$ ), with $|G|=\sqrt{G_{x}^{2}+G_{y}^{2}}=D G_{0}$ (a shell of RLVs), are given by the solutions to the following system of two nonlinear equations for $R$ and $\alpha$

$$
\begin{gathered}
G_{x} \cos \alpha+G_{y} \sin \alpha=\frac{2 \pi n}{R}, \\
G_{y} \cos \alpha-G_{x} \sin \alpha=\frac{2 \pi(2 k-n)}{\sqrt{3} R} .
\end{gathered}
$$

After some tortuous algebra, the solution to this system of equations becomes, ${ }^{19}$

$$
\begin{gathered}
R=\sqrt{\frac{n^{2}+k^{2}+n k}{a^{2}+b^{2}+a b}}, \\
\alpha=\arctan \left[\frac{-\sqrt{3} n G_{y}-(2 k+n) G_{x}}{-\sqrt{3} n G_{x}+(2 k+n) G_{y}}\right] .
\end{gathered}
$$

One can clearly distinguish $R$ as being an expression of type $R=\sqrt{\frac{N_{s u b}}{N_{a d s}}}$, which leads to the following Lemma.

Lemma 1. The Fourier term with RLVs of length $D G_{0}$ contributes to the energy of commensurate monolayer with $N_{a d s}=D^{2}$ particles per unit cell rotated by the angle $\alpha$ (given
TABLE I. Illustration of an algorithm for generation of a sequence of Fourier terms that contribute for a rigid hexagonal monolayer with $N_{a d s}=m$ particles. First, write down the hexagonal number sequence (first column) and the first Fourier term corresponding to each hexagonal number. Multiply $m$ by a successively larger number from the hexagonal number sequence and add it to the Fourier sequence. Repeat this procedure until the desired cutoff of the Fourier series.

\begin{tabular}{lcc}
\hline \hline$m=a^{2}+b^{2}+a b$ & First Fourier term & Fourier sequence \\
\hline 1 & 1 & All \\
3 & 2 & $2+5+6+10+\cdots$ \\
4 & 3 & $3+6+8+13+\cdots$ \\
7 & 4 & $4+10+13+20+\cdots$ \\
9 & 5 & $5+12+15+24+\cdots$ \\
12 & 6 & $6+15+19+32+\cdots$ \\
13 & 7 & $7+17+21+\cdots$ \\
16 & 8 & $8+19+25+\cdots$ \\
19 & 9 & $9+22+\cdots$ \\
21 & 10 & $10+24+\cdots$ \\
25 & 11 & $\vdots$ \\
27 & 12 & \\
28 & 13 & \\
$\vdots$ & $\vdots$ & \\
\hline \hline
\end{tabular}

by the preceding equation) with respect to the substrate.

At this point we can generate a sequence of Fourier terms which correspond to a commensurate monolayer with $N_{a d s}$ particles. First, one needs to realize that the identity $\frac{N_{a d s, 1}}{N_{s u b, 1}}$ $=\frac{N_{a d s, 2}}{N_{s u b, 2}}$ implies that $N_{a d s, 2}=m N_{a d s, 1}$ and $N_{s u b, 2}=m N_{s u b, 1}$, where $m \in \mathcal{H}$. $N_{a d s, 2}$ and $N_{s u b, 2}$ belong to $\mathcal{H}$, because of the closure property. Therefore, for any given monolayer-surface system, all expanded structures with same coverage can be obtained from multiplication by a successively larger $m \in \mathcal{H}$. Let us consider the trivial case of $N_{a d s}=1$ monolayer placed onto a substrate with $N_{\text {sub }}$ particles. Using the above lemma, the Fourier term with the first shell of RLVs contributes in this case. Now, expand the unit cells by multiplying both $N_{a d s}$ and $N_{\text {sub }}$ by $m \in \mathcal{H}$. In this case, the Fourier term associated with $m N_{a d s}$ contributes as well. This procedure can be repeated for any $m \in \mathcal{H}$. Therefore, we have shown that all Fourier terms contribute for $N_{a d s}=1$. Now, take a monolayer with $N_{a d s}=3$ and arbitrary $N_{s u b}$. In this case, the Fourier term associated with the second shell of RLVs contributes. Repeat the procedure stated above. It can be seen that for $N_{a d s}=3$, the Fourier terms of RLV shells 2, 5, 6, 10, .., contribute. Similarly, for $N_{a d s}=4$, the terms of RLV shells 3, 6, 8, 13, .., contribute. Such construction gives a hint on an algorithm to generate all Fourier terms that play a role for the rigid hexagonal commensurate structure with a given number of particles. See Table I for an illustration of such an algorithm. We will call $\mathcal{H}_{N}$ the set formed by all Fourier terms $\mathcal{F}_{i}$ which are conserved for a monolayer with $N_{a d s}=N$ (column 3 in Table I). Clearly, $\mathcal{H}_{N} \subseteq \mathcal{H}$.

Note that we did not mention anything about the monolayer rotation angle $\alpha$ in the above discussion. The expanded 
TABLE II. First seven Fourier coefficients (in $\mathrm{kcal} / \mathrm{mol}$ ) for iodine adsorbed on the $\operatorname{Pt}(111)$ surface. The coefficients are fitted to the unequal-sphere packing adsorption energies taken from Ref. 24 (with negative sign).

\begin{tabular}{ccccccc}
\hline \hline$V_{G_{0}}$ & $V_{\sqrt{3} G_{0}}$ & $V_{2 G_{0}}$ & $V_{\sqrt{7} G_{0}}$ & $V_{3 G_{0}}$ & $V_{2 \sqrt{3} G_{0}}$ & $V_{\sqrt{13} G_{0}}$ \\
\hline 0.9969 & -0.3379 & -0.1358 & 0.1421 & 0.0146 & -0.0710 & -0.0276 \\
\hline \hline
\end{tabular}

monolayers had exactly the same angle as the primitive ones. However, given a monolayer with $N_{a d s}$ particles, the rotation angle $\alpha$ is not unique. This is better illustrated with an example. Let us take a system with $N_{a d s}=21$ and $N_{s u b}=49$. Depending on the $a$ and $b$ values used to generate $N_{a d s}$, the rotation angle $\alpha$ can take two different values according to Eq. (6): $10.893^{\circ}$ and $27.320^{\circ}$. The former corresponds to a reduced $N_{a d s}=3$ and $N_{s u b}=7$ structure with energy given by $\mathcal{H}_{3}$, whereas the latter structure is given by $N_{a d s}=21$ and $N_{\text {sub }}=49$ with energy equal to $\mathcal{H}_{10}$.

Finally, the translational ground states $\left(x_{0}, y_{0}\right)$ for a commensurate hexagonal monolayer composed of $N$ atoms are obtained as solution to

$$
\min \sum_{\mathcal{F}_{i} \in \mathcal{H}_{N}} V_{G_{i}} \exp \left[-\imath\left(G_{i, x} x_{0}+G_{i, y} y_{0}\right)\right] .
$$

Note that only at this point one needs the exact values of Fourier amplitudes $V_{G}$ to solve the equation and obtain the stable $\left(x_{0}, y_{0}\right)$ pairs. The theory above Eq. (7) is exact for arbitrary values of $V_{G}$ amplitudes. Clearly, the relative magnitudes of the $V_{G}$ 's are also important to speculate about the probability of existence of a monolayer with a certain symmetry.

\section{APPLICATION OF THE THEORY TO REAL SYSTEMS}

The first impact of the presented theory on the experimental results is that one would expect to observe a perfect devil's staircase $e^{20}$ if the Fourier amplitudes of the atom-surface potential showed a strictly decreasing behavior. Actually, experimental observations in the $\operatorname{Ar}-\operatorname{Pt}(111)$ system seem to support this proposition. ${ }^{21}$ Another example of such behavior is the $\mathrm{I}-\mathrm{Pt}(111)$ system, where phase transitions from $(\sqrt{3}$ $\times \sqrt{3}) R 30^{\circ}\left(N_{a d s}=1, N_{s u b}=3\right)$ to $(\sqrt{7} \times \sqrt{7}) R 19.1^{\circ} \quad\left(N_{a d s}=3\right.$, $\left.N_{\text {sub }}=7\right)$ to $(3 \times 3)\left(N_{a d s}=4, N_{\text {sub }}=9\right)$ structures have been observed. ${ }^{22}$ However, many other examples exist in the experimental literature on well-ordered commensurate monolayers where no such defined sequence was found. In part, this could be due to the influence of the attractive lateral interactions in the monolayer, which confine it to a certain restricted coverage range. Nevertheless, an unusual behavior in the Fourier coefficients for complex adsorption systems could also make higher-order commensurate structures more stable than the lower-order ones. For instance, the Fourier terms for the first three RLV shells $\left(|G|=G_{0}, \sqrt{3} G_{0}, 2 G_{0}\right)$ possess corrugation $\left[\max \left(\mathcal{F}_{i}\right)-\min \left(\mathcal{F}_{i}\right)\right]$ equal to 9 , but the Fourier term for $|G|=\sqrt{7} G_{0}$ has corrugation equal to 18 . This fact is related to the number of solutions to Eq. (1) for a particular $m$. Therefore, if $\left|V_{\sqrt{7} G_{0}}\right|>0.5\left|V_{2 G_{0}}\right|$, the commensurate structure with $N_{a d s}=7$ would be more stable than the lower-order one with $N_{a d s}=4$.
Now, we proceed to a thorough analysis of the Fourier expansion of the I-Pt(111) system, where a very precise model of the atom-surface interaction is available, ${ }^{23,24}$ which allows us to fit a large number of Fourier amplitudes. Furthermore, a wide variety of experiments have been performed on this system and the commensurate structures are identified and described in great detail. ${ }^{22}$ The first seven I-Pt(111) Fourier coefficients are listed in Table II. First of all, it is clear that the Fourier series does not show strictly decreasing behavior. There are at least three sign changes in the coefficients, and particularly $\left|V_{2 \sqrt{3} G_{0}}\right|$ is almost five times larger than $\left|V_{3 G_{0}}\right|$. The fit of coefficients higher than $V_{\sqrt{13}} G_{0}$ showed that the series eventually converges. Many experimental studies of the I-Pt(111) system show ${ }^{5,25,26}$ that there exist two different rigid translational possibilities in the $(3$ $\times 3)$ cell: sym and asym, both characterized by a perfect hexagonal arrangement inside the unit cell. From our theory, the translational energy $E_{(3 \times 3)}(\mathbf{r})=\sum_{\mathcal{F}_{i} \in \mathcal{H}_{4}} \mathcal{F}_{i}$ for $i \leqslant 7$ is $\mathcal{F}_{3}(\mathbf{r})+\mathcal{F}_{6}(\mathbf{r}) . E_{(3 \times 3)}(\mathbf{r})$ is depicted in Fig. 2 . Using the second derivative test, one can see that for $V_{2 G_{0}}<0$ and $V_{2 \sqrt{3} G_{0}}<0$, the global minimum is at $(x, y)=(0,0)[\mathrm{sym}]$ and another local minimum is at $(x, y)=(0.25, \sqrt{3} / 12)$ [asym]. The models for these two minima are depicted in Figs. 1(a) and $1(\mathrm{~b})$ of Ref. 24. For $\mathcal{F}_{3}(\mathbf{r})$ alone with $V_{2 G_{0}}<0$, the (3 $\times 3)$-sym structure is the only possible minimum, and (3 $\times 3$ )-asym can become minimum exclusively because of the influence of the sixth Fourier term. Namely, its stability is determined by the inequality $\left|V_{2 \sqrt{3} G_{0}}\right|>0.16\left|V_{2 G_{0}}\right|$, which is certainly fulfilled in the case of $\mathrm{I}-\mathrm{Pt}(111)$. Another interesting issue is that the structures $(\sqrt{19} \times \sqrt{19}) R 23.4^{\circ}\left(N_{a d s}=7\right.$ and $\left.N_{s u b}=16\right)$ and $(4 \times 4)\left(N_{a d s}=7\right.$ and $\left.N_{s u b}=19\right)$ with coverage $\theta=0.368$ and $\theta=0.438$, respectively, which are stabilized by

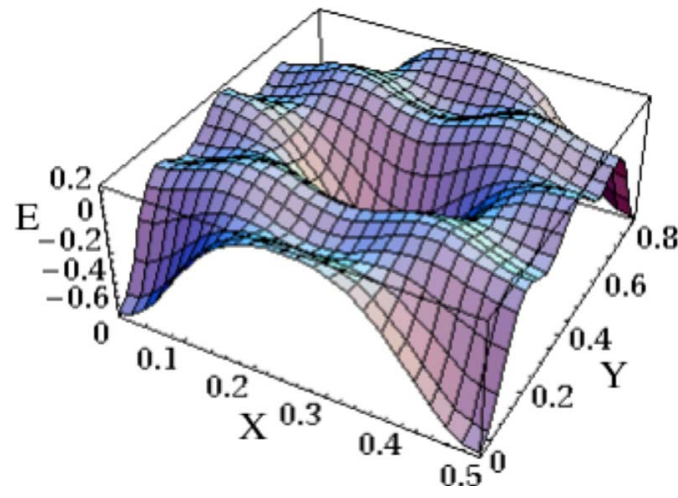

FIG. 2. (Color online) Plot of $E_{(3 \times 3)}(\mathbf{r})=V_{2 G_{0}} \mathcal{F}_{3}(\mathbf{r})$ $+V_{2 \sqrt{3} G_{0}} \mathcal{F}_{6}(\mathbf{r})$. The values of the Fourier coefficients are taken from Table II. The $X$ and $Y$ axes are in units of substrate lattice constant $((l=1)$ and $E$ is in $\mathrm{kcal} / \mathrm{mol}$. 


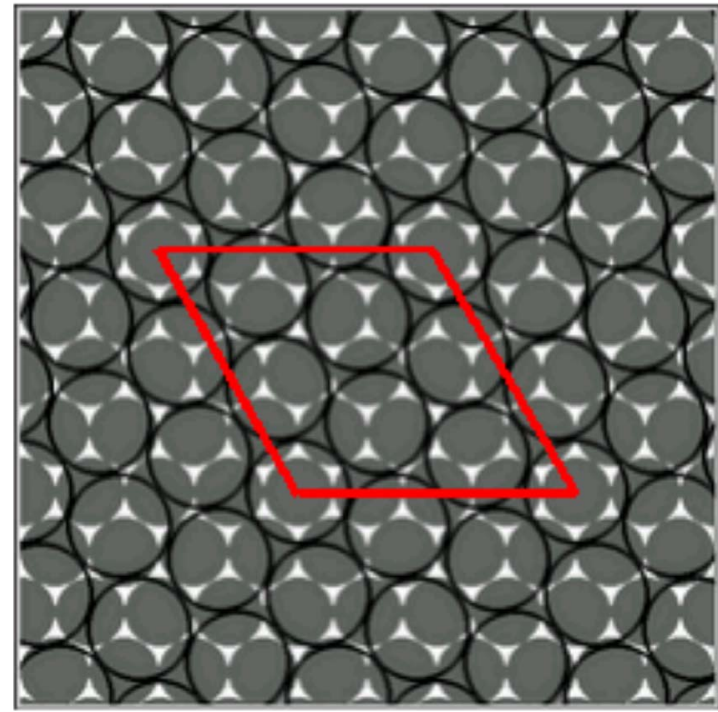

FIG. 3. (Color online) Model of the $(4 \times 4)$ structure with $\theta$ $=7 / 19$. The filled gray circles indicate the substrate atoms $[(111)$ surface], while bigger black circles show adsorbed monolayer atoms. The unit cell is marked by a red (dark gray) rhomb. The picture was generated by the ALSA software, see Ref. 8 .

$\mathcal{F}_{4}$, have never been observed. Their coverage lies within the experimental range and the value of $V_{\sqrt{7} G_{0}}$ indicates that they should be stable. The nonexistence of the $(\sqrt{19}$ $\times \sqrt{19}) R 23.4^{\circ}$ structure could be attributed, in part, to possible attractive lateral interactions, which drive the I-Pt(111) system directly from $(\sqrt{3} \times \sqrt{3}) R 30^{\circ}(\theta=0.333)$ to $(\sqrt{7}$ $\times \sqrt{7}) R 19.1^{\circ}(\theta=0.428)$. However, the $(4 \times 4)$ structure $(\theta$ $=0.4375)$, shown in Fig. 3, lies exactly in between $(\sqrt{7}$ $\times \sqrt{7}) R 19.1^{\circ}$ and $(3 \times 3) \quad(\theta=0.444)$ structures, both observed in experiments for the I-Pt(111) system. ${ }^{22}$ In such small coverage range $(0.428 \leqslant \theta \leqslant 0.444)$, the lateral interactions could be assumed constant, and the stability of commensurate hexagonal structures would be determined by the corresponding atom-surface Fourier terms. In this picture, the $(4 \times 4)$ structure is definitely stable for the I-Pt(111) system. However, as shown in Ref. 15 , the above structure lies outside of the simple linear trajectory connecting $(\sqrt{3}$ $\times \sqrt{3}) R 30^{\circ}$ to $(\sqrt{7} \times \sqrt{7}) R 19.1^{\circ}$ to $(3 \times 3)$. These data support the view that a very special experimental preparation procedure is required to locate the $(4 \times 4)$ structure. This fact, along with the discovery of the novel $(3 \times 3)$-zigzag structure in Ref. 24, points to the need of additional thorough experimental investigations of the I-Pt(111) system.

\section{DISCUSSION}

It is important to stress the main point of this work once again: the knowledge of the atom-surface potential alone is sufficient to predict all possible rigid commensurate ground states for monolayers on surfaces.

Once one joins the algorithm for monolayer construction with the formula for $R$ and $\alpha$ in Eq. (6), all commensurate monolayers, for which a sequence of Fourier terms contrib- ute, can be generated by following the procedure described above. This algorithm could be of great use for systematic density functional theory (DFT) studies, when one wants to sample a variety of commensurate cells and extract details of the potential energy surface (i.e., first-principles determination of the lateral interactions in the monolayer). Furthermore, the knowledge of the $V_{G}$ amplitudes, whose values could be readily obtained in DFT calculations, allows one to exactly predict the translational $\left(x_{0}, y_{0}\right)$ ground states in commensurate unit cells.

The approach of this study is also useful for interpretation and prediction of stable monolayer-surface structures in experiments. Namely, when a certain commensurate structure has been identified, it is worth looking for other commensurate structures which are close in coverage or possess higher symmetry. One expects lower energy structures to have higher symmetry, although this would require that the Fourier amplitudes be strictly decaying. Although this seems to be the case for some physisorption systems, ${ }^{17,18}$ many examples of higher-order commensurate structures exist in the experimental literature on atomic and molecular adsorption, 5,9,11,13,21,22,27-29 where the stability should be mainly determined by the contribution of the high-order Fourier terms. Furthermore, the knowledge of the Fourier amplitudes for any particular system (i.e., obtained from DFT calculations) could allow better interpretation of the translational symmetry of commensurate monolayers in addition to the fitting of the LEED spectra.

Throughout this work, it is assumed that the Fourier amplitudes of the atom-surface potential remain constant for any value of coverage $\theta$. However, experimentally, it has been shown that the most stable adsorption site could depend on $\theta,{ }^{30}$ indicating that the Fourier amplitudes are not constant, at least for some complex adsorption systems. This effect is most probably due to the coupling of isolated atomsurface interaction and the indirect substrate induced forces. However, even in such cases, one could interpolate between the different values of the Fourier amplitudes and analyze the commensurate ground states in terms of $V_{G}(\theta)$.

\section{SUMMARY}

In conclusion, a procedure has been presented which can be used to generate all rigid hexagonal commensurate structures of a monolayer adsorbed on the (111) surface. Each of these monolayers is associated with a well-defined sequence of the atom-surface potential Fourier terms, and its translational energy can be simply written as a sum over this sequence. Unfortunately, first-principles description of the atom-surface potential is available for very few systems. Hopefully, this work will stimulate further research on the precise description of such interactions.

The theory presented in this work could probably be extended to treat coincident organic molecule epitaxy, ${ }^{28}$ where the molecule-surface potential has been shown to dominate the structure formation. ${ }^{28,31}$ Another possible application concerns the structure of intercalated crystals, where the 
three-dimensional Fourier expansion describes the field felt by a foreign element introduced inside of a crystal. ${ }^{32}$

The computer codes for generating all commensurate structures for a given coverage range and the Fourier sequence associated with each structure on (111) and (100) surfaces are available upon request from the author.

\section{ACKNOWLEDGMENTS}

The author wishes to express gratitude to Marcelo Galván for fruitful discussions, Adolfo Torres Cházaro and Mikhail Tkatchenko for help with number theory issues, and CONACYT/Mexico for financial support. *atkatchenko@gmail.com

${ }^{1}$ G. A. Somorjai, Introduction to Surface Chemistry and Catalysis (Wiley, New York, 1994).

${ }^{2}$ M. A. van Hove and S. Y. Tong, Surface Crystallography by LEED (Springer, Berlin, 1979).

${ }^{3}$ M. A. van Hove, W. H. Weinberg, and C.-M. Chan, Low Energy Electron Diffraction (Springer-Verlag, Berlin, 1986).

${ }^{4}$ R. Bennewitz, J. N. Crain, A. Kirakosian, J.-L. Lin, J. L. McChesney, D. Y. Petrovykh, and F. J. Himpsel, Nanotechnology 13, 499 (2002).

${ }^{5}$ B. C. Schardt, S. L. Yau, and F. Rinaldi, Science 243, 1050 (1989).

${ }^{6}$ R. I. Masel, Principles of Adsorption and Reaction on Solid Surfaces (Wiley, New York, 1996).

${ }^{7}$ In what follows, we will use the word "atomic" to refer to atomic and molecular adsorption systems without distinction.

${ }^{8}$ A. Tkatchenko and N. Batina, J. Chem. Phys. 122, 094705 (2005).

${ }^{9}$ R. D. Diehl, T. Seyller, M. Caragiu, G. S. Leatherman, N. Ferralis, K. Pussi, P. Kaukasoina, and M. Lindroos, J. Phys.: Condens. Matter 16, 2839 (2004).

${ }^{10}$ Although the primitive symmetry of the monolayers treated in this work is triangular, the triangular symmetry is a subset of the hexagonal one, and we use the word "hexagonal" for consistent naming of the substrate and adsorbate symmetries.

${ }^{11}$ B. Narloch and D. Menzel, Surf. Sci. 412, 562 (1998).

${ }^{12}$ T. Seyller, M. Caragiu, R. D. Diehl, P. Kaukasoina, and M. Lindroos, Phys. Rev. B 60, 11084 (1999).

${ }^{13}$ M. Saidy, K. A. R. Mitchell, S. A. Furman, M. Labayen, and D. A. Harrington, Surf. Rev. Lett. 6, 871 (1999).

${ }^{14}$ K. Pussi, J. Smerdon, N. Ferralis, M. Lindroos, R. McGrath, and R. D. Diehl, Surf. Sci. 548, 157 (2004).
${ }^{15}$ A. Tkatchenko, Phys. Rev. B 74, 035428 (2006).

${ }^{16}$ H. L. Keng, Introduction to Number Theory (Springer-Verlag, Berlin, 1982).

${ }^{17}$ W. A. Steele, Surf. Sci. 36, 317 (1973).

${ }^{18}$ L. W. Bruch, M. W. Cole, and E. Zaremba, Physical Adsorption: Forces and Phenomena (Oxford University Press, New York, 1997).

${ }^{19}$ In order to get Eq. (6) from Eq. (5), one needs to substitute $n$ by $-n$, without affecting the outcome.

${ }^{20}$ B. B. Mandelbrot, The Fractal Geometry of Nature (Freeman, New York, 1982).

${ }^{21}$ P. Zeppenfeld, U. Becher, K. Kern, and G. Comsa, Phys. Rev. B 45, 5179 (1992).

${ }^{22}$ O. M. Magnussen, Chem. Rev. (Washington, D.C.) 102, 679 (2002).

${ }^{23}$ A. Tkatchenko and N. Batina, Phys. Rev. B 70, 195403 (2004).

${ }^{24}$ A. Tkatchenko, N. Batina, and M. Galvan, Phys. Rev. Lett. 97, 036102 (2006).

${ }^{25}$ S. C. Chang, S. L. Yau, B. C. Schardt, and M. J. Weaver, J. Phys. Chem. 95, 4787 (1991).

${ }^{26}$ J. Inukai, Y. Osawa, M. Wakisaka, K. Sashikata, Y.-G. Kim, and K. Itaya, J. Phys. Chem. B 102, 3498 (1998).

${ }^{27}$ I. Villegas and M. J. Weaver, J. Chem. Phys. 101, 1648 (1994).

${ }^{28}$ D. E. Hooks, T. Fritz, and M. D. Ward, Adv. Mater. (Weinheim, Ger.) 13, 227 (2001).

${ }^{29}$ A. Lagutchev, G. Q. Lu, T. Takeshita, D. D. Dlott, and A. Wieckowski, J. Chem. Phys. 125, 154705 (2006).

${ }^{30}$ R. D. Diehl and R. McGrath, J. Phys.: Condens. Matter 9, 951 (1997).

${ }^{31}$ S. C. B. Mannsfeld and T. Fritz, Phys. Rev. B 69, 075416 (2004).

${ }^{32}$ A. Sütő, Phys. Rev. Lett. 95, 265501 (2005). 